\title{
Cervical evidement: classification and nomenclature
}

\author{
Advanced Center of Diagnostics Head and Neck, \\ Complexo Hospitalar Heliópolis - São Paulo, Brazil
}


ervical evidement is a surgery whose primary objective is the control of the regional lymphatic metastasis of primitive cancer in the region of the head and neck. The most important historical reference comes from CRILE (J Am Med Assoc 47:1780-88, 1906) who propose the same surgery principle for cancer of the head and neck region as that proposed by Halstead for breast cancer, that is, the primary lesion should be removed together with the local lymph nodes. This surgery is known even nowadays as radical cervical evidement in analogy with radical mastectomy which includes removal of a breast with the tumor and the local lymph nodes.

Taking the surgery of Crile as a reference, many variations have been proposed as much to reduce the morbidity (through reduction of the number of nonlymphatic structures removed ) as to change cervical evidement into minor surgery (by selecting some lymphatic chains to be deleted). In this direction the works of SUAREZ (Rev Otorinolaringol 23:93-99, 1963), BOCCA (J Laryngol 80:831-38,1966) e JESSE, BALLENTYNE \& and LARSON (Am J Surg 136:516-

Address for correspondence:

Marcos Brasilino de Carvalho

Praca Amadeu Amaral, 47 / Cj. 82

Sao Paulo - SP - Brasil - CEP 01327-010
19, 1978) and BALLENTYNE (Curr Probl Cancer 9:334,1985 ) are fundamental. The first two excised the same lymphatic chains as traditional evidement but conserved the submandibular gland, the sternocleidomastoid muscle, the internal jugular vein and the accessory spinal nerve. This surgery was called functional cervical evidement. BALLANTYNE also conserved those non-lymphatic structures but his surgery did not extend to the inferior $2 /$ 3 of the spinal nerve chain nor to the external third of the supraclavicular region. This surgery received the name of modified cervical evidement. LINGEMAN and colleagues (Ann Otol 86:737-44, 1977) describe a technique of cervical evidement similar to that of SUAREZ and that of BOCCA but they call it conservative cervical evidement. This technique contrasts itself with the so-called radical cervical evidement. The term radical has been employed in variable ways by various authors; ranging from a synonym for surgery that removes all the neoplasia ( as opposed to the palliative) to the idea of total, or complete (as opposed to partial or selective). Because it makes comprehension of this classification more complicated, the word radical should be avoided when designating a determined type of evidement.

The classification of different types of evidement has recently deserved the attention of authors responsible for important registration of clinical data, but they have not arrived at a consensus mainly because they propose that the denomination of the surgery include too much detail as to which structures are conserved or not (BYERS: 
Am J Surg 150:215-22, 1985: O'BRIEN and col.: Am J Surg 153:310-16, 1987; SUEN \& GOEPFERT, 9:75-77, 1987; ROBBINS and col.: Arch Otolaryngol/Head \& Neck Surg 117:601-05, 1991).

A fundamental rule by which a classification should be adopted is that it be based on logical principals and be easily understood. With respect to cervical evidements the most reasonable approach would be to use as a point of reference the lymphatic and non-lymphatic structures involved in the surgery. In this way, with respect to the removed lymphatic chains, the evidement would be complete (or total) or partial (or selective). The partial evidements have been classified according with regard to the included chains, and has not been a source of controversy. With regard to non-lymphatic elements, whether conserved or not, in the denomination of complete or partial cervical evidement the structures that were saved would be mentioned, for ex.: "complete cervical evidement with conservation of the sternocleidomastoid muscle and the accessory spinal nerve"; "complete bilateral cervical evidement with conservation of the right internal jugular vein and the accessory spinal nerves".

The qualifying terms conservative, functional, classic, modified and radical should be abandoned, as their usage demands an accompanying explanation as to the intended meaning interpreted by the author, which makes standardization difficult.
Finally, some words about the term "lymphadenectomy". Under this denomination the authors have referred to the most minor surgery possible on the lymphatic system which is the extraction of a lymph node. Even today we can find references in the Index Medicus to lymphadenopathy and lymphadenectomy. The Nomina Anatomica of 1895 (Basileia) substituted the Greek term ganglion (which means gland) for the denomination of lymph gland. In 1935, in Jena the Nomina Anatomica definitively adopted the term lymph node as the most appropriate and as one of almost unanimous acceptance among different authors.

Inexplicably the idea of a lymph gland persists in the terminology related to surgery and lymph node pathology . The expressions lymphadenitis, lymphadenectomy and lymphadenopathy are commonly seen in scientific publications. Everyone knows that the composition element aden is from the original Greek word aden which means gland, and from there we have adenoma and adenocarcinoma to designate benign and malignant tumors derived from the glandular tissue. Therefore, if we have abandoned the expression lymph gland and prefer the term lymph node, then for etymological coherence we should use the terms lymphonoditis, lymphonodopathy and lymphonodectomy when referring to, respectively, an inflammation, a pathology, and lymph node surgery. 